\title{
SUSTENTABILIDADE DA INVESTIGAÇÃO EM EDUCAÇÃO: DA CONCEPÇÃO À IMPLEMENTAÇÃO DE UM REFERENCIAL
}

\author{
SUSTAINABILITY OF RESEARCH IN EDUCATION: \\ FROM CONCEPTION TO IMPLEMENTATION OF A FRAMEWORK
}

SUSTENTABILIDAD DE LA INVESTIGACIÓN EN EDUCACIÓN: DE LA CONCEPCIÓN A LA IMPLEMENTACIÓN DE UN REFERENTE

\begin{abstract}
Cecília Guerra
Centro de Investigação "Didática e Tecnologia na Formação de Formadores” da Universidade de Aveiro - Portugal
\end{abstract}

\begin{abstract}
Resumo: Este artigo pretende apresentar um recorte do processo metodológico referente ao desenvolvimento de um referencial para a promoção da sustentabilidade de investigação em educação. Este produto decorre de um estudo de investigação e desenvolvimento (I\&D), envolvendo a colaboração com diversos agentes-chave do ensino superior português (investigadores e docentes universitários) no desenho, implementação e avaliação da qualidade de estratégias e instrumentos promotoras da sustentabilidade de inovações educativas resultantes da investigação. Os dados foram recolhidos através da aplicação de questionários, realização de entrevistas e observação participante da investigadora do projeto. A análise de conteúdo dos documentos produzidos pelos participantes (ex. artigos científicos produzidos) e a análise dos registos de observação do trabalho desenvolvido na rede (observação participante da investigadora do estudo) é um processo contínuo, com ciclos de reflexão-ação para ajudar a identificar as potencialidades, constrangimentos e definir melhorias para produzir a segunda versão do referencial. Pretende-se, por fim, informar (e direcionar) a sustentabilidade de inovações educativas desenvolvidas a partir de investigação educacional financiada.
\end{abstract}

Palavras chave: sustentabilidade da investigação; inovações educativas; investigação \& desenvolvimento.

\begin{abstract}
This article aims to present part of the methodological process related to the development of a benchmark for the promotion of sustainability of research in education. This product derives from a research and development (R\&D) study, involving collaboration with several key agents of Portuguese higher education (researchers and university lecturers) in the design, implementation and quality assessment of strategies and instruments promoting the sustainability of educational innovations resulting from research. Data were collected through the application of questionnaires, interviews and participant observation by the project researcher. The content analysis of the documents produced by the participants (e.g. scientific articles produced) and the analysis of the observation records of the work developed in the network (participant observation by the study researcher) is an ongoing process, with cycles of reflection-action to help identify potentials, constraints and define improvements to produce the second version of the referential. It is intended, finally, to inform (and direct) the sustainability of educational innovations developed from funded educational research.
\end{abstract}

Keywords: sustainability of research; educational innovations; research \& development. 
Resumen: Este artículo pretende presentar parte del proceso metodológico relacionado con el desarrollo de un punto de referencia para la promoción de la sostenibilidad de la investigación en educación. Este producto es el resultado de un estudio de investigación y desarrollo (I+D), que implica la colaboración con varios agentes clave de la educación superior portuguesa (investigadores y profesores universitarios) en el diseño, la aplicación y la evaluación de la calidad de las estrategias e instrumentos que promueven la sostenibilidad de las innovaciones educativas resultantes de la investigación. Los datos se recogieron mediante cuestionarios, entrevistas y observación participante por parte del investigador del proyecto. El análisis de contenido de los documentos producidos por los participantes (por ejemplo, los artículos científicos producidos) y el análisis de los registros de observación del trabajo desarrollado en la red (observación participante del investigador del estudio) es un proceso continuo, con ciclos de reflexiónacción que ayudan a identificar los potenciales, las limitaciones y a definir las mejoras para producir la segunda versión del benchmark. Se pretende, por último, informar (y dirigir) la sostenibilidad de las innovaciones educativas desarrolladas a partir de la investigación educativa financiada.

Palabas clave: sostenibilidad de la investigación; innovaciones educativas; investigación y desarrollo.

\section{Introdução}

A sustentabilidade da investigação é uma condição essencial para mobilização do conhecimento científico produzido nos projetos, sendo necessário um compromisso ético dos investigadores com a sua promoção nas diversas esferas da sociedade (ex. académica, empresarial, política). Tal desiderato tem vindo a ser inscrito nas agendas da comunidade científica da investigação em educação e pelas agências de financiamento da Ciência e Tecnologia (European Commission, 2020). Mas, na prática, que esforços concretos, mensuráveis e sustentáveis estarão a ser encetados de modo a assegurar que os resultados da investigação em educação (sobretudo aquela que é financiada) estão a ser, efetivamente, promovidos deliberada, explícita e sistematicamente pelos os agentes-chave de instituições científicas (ex. instituições de ensino superior e centros de investigação)?

Dada a sua relevância e face a tais questionamentos, neste artigo pretende-se apresentar o relato de um estudo (ainda em desenvolvimento) que almeja aprofundar aspetos teóricos e metodológicos relacionados com o processo de promoção da sustentabilidade da investigação em educação. Em particular, neste projeto pretende-se investigar em que medida os projetos de investigação financiados que se debruçam sobre o estudo de problemáticas educacionais significativas para diferentes atores (ex. docentes e estudantes) e sistemas (ex. instituições de ensino superior) concorrem, ou não, para a sustentabilidade da investigação, após o término do período de financiamento.

A problemática de investigação não tem (nem pode ter!) uma resposta direta e simples, pois tem implicado um percurso metodológico complexo, labiríntico, desafiante, característico da investigação qualitativa (Ribeiro, Guerra, \& Sá, 2016), recorrendo a metodologias de investigação diversificadas, oriundas de outras áreas científicas, como o Design. É um projeto 
que assenta numa 'rede epistémica' de conhecimento científico que recorre (mas também contribui) para o avanço da investigação na área da Didática e Tecnologia Educativa na Formação de Formadores.

Para contextualizar este projeto, é necessário explicar a sua origem, uma vez que resulta da participação da autora deste artigo - enquanto jovem investigadora doutorada - em dois projetos focados no ensino superior português.

O primeiro projeto, coordenado por Helena Pedrosa-de-Jesus (CIDTFF-UA) entre setembro de 2012 e agosto de 2015, intitulou-se "Estudo do desenvolvimento académico na universidade através de abordagens inovadoras de ensino, avaliação e feedback" (PTDC/CPE CED/117516/2010) ${ }^{1}$. O projeto tinha como finalidade produzir conhecimento sobre o desenvolvimento académico de docentes do ensino superior através do desenho e ensaio de práticas inovadoras de ensino, aprendizagem, avaliação e feedback (EAAF), com o envolvimento e contributo de um grupo de docentes universitários de Biologia. Na sua essência, os resultados deste projeto demonstraram que foi possível encontrar respostas para questões académicas de EAAF, através de observações em sala de aula e da promoção de investigaçãoação e da reflexão académica dos docentes envolvidos. A caracterização do estudo, bem como alguns dos resultados podem ser consultados em (Guerra, Correia, \& Pedrosa-de-Jesus, 2014; Guerra, Pedrosa-de-Jesus, Correia, Cunha, Almeida, \& Watts, 2015; Pedrosa-de-Jesus, Correia, Gonçalves, Almeida, Cunha, Moreira, da Silva Lopes, Guerra, C., \& Watts, 2015; Pedrosa-deJesus, Guerra, \& Watts, 2017; Pedrosa-de-Jesus, Guerra, \& Watts, 2018; Pedrosa-de-Jesus, Moreira, da Silva Lopes, Guerra, C., \& Watts, 2018; Pedrosa-de-Jesus., Guerra, \& Watts, 2019).

O segundo projeto, resultante de uma bolsa de pós-doutoramento sob orientação científica de Nilza Costa (CIDTFF-UA), decorreu entre setembro de 2015 e dezembro de 2018, e intitulou-se "Sustentabilidade dos resultados de investigação na inovação educativa no contexto universitário português" (SFRH/BPD/103497/2014). O estudo teve como finalidade estudar a sustentabilidade dos resultados emergentes da investigação em educação centrada na promoção da inovação de EAAF em cursos de Engenharias e Ciências de instituições de ensino superior publico português (IESPP). Deste modo, foram recolhidos e analisados dados que permitiram caraterizar os projetos de investigação educacional selecionados, face aos critérios definidos, no sentido de averiguar em que medida estes consideraram a sustentabilidade dos seus resultados desde a sua conceção e compreender as dinâmicas ocorridas nas práticas de EAAF, e nos seus atores, face às inovações ocorridas durante a implementação dos projetos e após o término destes, bem como as razões subjacentes às mesmas (Guerra, \& Costa, 2016a, 2016b;

\footnotetext{
${ }^{1}$ http://edaun.web.ua.pt
} 
2018; Guerra, Franco, \& Seabra, 2018).

Ambos os projetos ancoram-se no paradigma socio-crítico, seguindo uma metodologia mista (no geral, mais expressiva na vertente qualitativa do que na quantitativa). Para tal, adotou uma metodologia de investigação de cariz qualitativo (Bogdan, \& Biklen, 1994), situando-se num paradigma fenomenológico-interpretativo (Coutinho, 2014), enquadrando-se no plano investigativo da investigação-ação e do tipo estudo de caso coletivo (Creswell, Hanson, Clark Plano, \& Morales, 2007), uma vez que se debruçou sobre um conjunto de casos individualmente (ex. as práticas académicas dos docentes de Biologia (projeto 1) ou nos projetos financiados (projeto 2), com vista a ampliar a compreensão sobre o desenvolvimento e a sustentabilidade dos resultados emergentes da investigação financiada centrada na promoção da inovação educativa do IESPP. Os dados recolhidos foram analisados através da técnica de análise de conteúdo (Neuendorf, 2018), com recurso ao software de análise qualitativa webqda.

São destes pontos de partida investigativos que surge o presente projeto intitulado "Desenvolvimento e sustentabilidade de inovações educativas no ensino universitário português: investigar para orientar as políticas de formação e de investigação das instituições". O projeto teve a sua génese num plano de trabalhos entregue à Reitoria da Universidade de Aveiro em janeiro de 2019, para a concretização do contrato de investigadora (REF. 008 - 88ARH/201), com a finalidade de investigar e desenvolver soluções sobre processos promotores da sustentabilidade da investigação em educação. O projeto pretende contribuir para um pensamento reflexivo sobre as seguintes questões de investigação:

1) Que estratégias e instrumentos são considerados, pelas evidências da sua eficácia na literatura, mais favoráveis à promoção da sustentabilidade da investigação em educação?

2) Como promover a mobilização do conhecimento científico em investigação em educação, para uma maior articulação entre a investigação, a formação e a extensão à sociedade?

Todavia, face aos constantes desafios investigativos que fizeram (e ainda fazem) parte do quotidiano de uma 'jovem' investigadora em educação, rapidamente se constatou que o plano teria que sofrer alterações metodológicas. Este 'acontecimento' pode ser encarado como um processo inerente à metamorfose científica inerente à evolução de projetos de investigação em educação, em particular aqueles que dependem de estímulos individuais e coletivos por parte de diversos atores e sistemas educativos.

Neste artigo, pretende-se ilustrar, ainda que de forma resumida, as experiências realizadas no projeto de investigação, produtos catalisados e resultados alcançados com o foco na sustentabilidade da investigação em educação. 
Mais do que um mero inventário de atividades investigativas realizadas e instrumentos de recolha e análise de dados produzidos durante este projeto, ambiciona-se partilhar com a comunidade científica um percurso de investigação em educação, sustentado por uma persistente reflexão crítica da autora durante o projeto.

Por conseguinte, as eventuais lacunas que o artigo vier a revelar poderão resultar, não só do imprevisível processo de escrita académica (que se aperfeiçoa a cada instante), mas também do desejo de traduzir a riqueza dos principais procedimentos metodológicos, resultados alcançados e produtos científicos catalisados ao longo do projeto.

$\mathrm{O}$ artigo encontra-se estruturado em quatro partes. Na primeira parte apresenta-se o enquadramento teórico que sustenta o trabalho realizado, centrado na sustentabilidade da investigação em educação. Na segunda parte descreve-se a metodologia de investigação, técnicas e instrumentos de recolha e análise de dados desenvolvidos. Na terceira parte apresenta-se as o processo de desenvolvimento do referencial de Sustentabilidade da Investigação em Educação (rSIE). Por último, apresentam-se as considerações gerais do estudo, as suas limitações e o trabalho futuro.

\section{Referencial Teórico}

No ensino superior (ES), vários atores-chave têm vindo a desenvolver projetos de investigação em educação (Boshier, 2009; Hutchings, Huber, \& Ciccone, 2011; Laksov, 2019). No contexto de ES Público Português, Guerra, \& Costa (2016b; 2018) constataram que os projetos financiados se focaram, sobretudo, ao nível do desenvolvimento de estratégias de ensino, aprendizagem, avaliação, feedback (EAAF) e/ou recursos educativos de base tecnológica (ex. laboratórios virtuais remotos) em cursos de Ciências Exatas (ex. Física), Naturais (ex. Biologia) e, em número mais significativo, de Engenharia (ex. Mecânica).

Todavia, Guerra, \& Costa (2016b; 2018) revelaram que a avaliação das inovações resultantes de investigação em educação implementadas no ensino universitário (ex. o impacte nas aprendizagens dos estudantes de Química (Del Pino \& Cachapuz, 2008), continua a ser limitado, desconhecendo-se, na globalidade, qual é a sustentabilidade dos resultados da investigação financiada, sobretudo a médio e a longo prazo.

Tendo em linha de conta as recomendações da Comissão Europeia (2014), importa garantir o acesso aos resultados da investigação financiada, bem como averiguar a sua sustentabilidade após o término dos projetos. Porém, a maioria das inovações produzidas no contexto de projetos nacionais financiados, nem sempre tiveram a sustentabilidade desejada. Algumas razões foram identificadas no estudo de Guerra \& Costa $(2016 b ; 2018)$ para esta 
situação, em particular a 'inação' de atores-chave da educação para manter/adaptar e/ou sustentar o conhecimento científico gerado pelos projetos financiados no ensino superior, em particular: os políticos (que definem os currículos), os líderes institucionais (que apoiam a manutenção e/ou renovação dos projetos), os indivíduos (que participaram nos projetos e poderiam manter as inovações).

No mundo académico, vários autores têm vindo a estudar sobre o significado de "sustentabilidade da investigação", sua operacionalidade no âmbito dos projetos, e formas de avaliação (ex. Faber, Jorna, \& Van Engelen, 2009; Becan, et al, 2018; Sarriot, Ricca, Yourkavitch, \& Ryan, 2008; Scheirer, 2005; Scheirer, 2013; Schell, Luke, Schooley, Elliott, Herbers, Mueller, \& Bunger, 2013; Shediac-Rizkallah, \& Bone, 1998). O facto de não existir (ainda) uma discussão alargada na comunidade científica sobre o conceito e sua operacionalidade em investigação em educação no contexto do ES, tem dificultado a compreensão sobre as (inter)relações epistémicas que se estabelecem entre os fatores que influenciam a sustentabilidade da investigação (Guerra, \& Costa, 2016b; 2018, Guerra, Franco \& Seabra, 2021).

A sustentabilidade da inovação pode implicar a implementação parcial das práticas de ensino e aprendizagem desenvolvidas, a sua modificação e/ou a sua expansão em outros contextos. De forma breve, a Sustentabilidade da Investigação em Educação neste contexto pode ser encarada como Guerra (2021) propõe:

\begin{abstract}
"The sustainable use of pedagogical innovations developed in the scope of funded educational research is considered to be achieved when after a defined period of time, pedagogical innovations continue to be delivered in higher education, with or without adaptations, in the same academic scenery (e.g., course or curricular unit), with the aim of producing benefits for individuals (e.g., students' academic success and/or teachers' academic growth) and/or systems (e.g., institutions, courses, curricular units)." (p. 10)
\end{abstract}

Embora a promoção da sustentabilidade da investigação seja indispensável para diferentes atores e sistemas, a sustentabilidade da investigação exige da parte de diversos atoreschave no 'terreno' ações promotoras:

" ... the continuation of project components (e.g., the innovative practice developed), capacity building (e.g., human and financial resources), and continued benefits or outcomes in the same and/or new settings (e.g., new educational scenarios and participants)" (Costa \& Guerra, 2021, p. 117).

Destaca-se, ainda, o potencial da institucionalização da inovação através da incorporação desta pelos docentes e/ou políticas e/ou processos organizacionais (Fishman, Penuel, Hegedus, \& Roschelle, 2011). Fatores contextuais, como cenários sociopolíticos, influenciam as formas 
em que os projetos evoluem após a implementação e são sustentados no tempo em configurações do mundo real, especialmente se as ações foram originalmente experimentadas entre os participantes ou em circunstâncias não representativas.

Os projetos de estudo de métodos mistos são uma forma de estudar a sustentabilidade a investigação em educação, mas é crucial investir em mais estudos empíricos para avançar este campo do conhecimento científico (Costa \& Guerra, 2021, p. 117). Assim, é fundamental contribuir para a sistematização do conhecimento construído no âmbito da investigação em educação, bem como averiguar quais são os fatores que contribuem (ou não) para a sustentabilidade dos resultados obtidos com essa investigação (ex. inovações educativas desenvolvidas no ES).

Loh, Friedman, \& Burdick (2013) estudaram os fatores que suportam a sustentabilidade da investigação financiada, e que se relaciona com aspetos internos do projeto (ex. capacidade de liderança do coordenador do projeto e dinâmicas de trabalho dos elementos da equipa), e com os aspetos externos do projeto (ex. apoio institucional para a consecução dos objetivos do projeto).

Agências de financiamento da investigação, como a Comissão Europeia (2014, 2020), têm vindo a reforçar a necessidade de se investir na mobilização do conhecimento científico produzido no âmbito da investigação financiada. Também autores da investigação educacional (Bennet et al., 2007; Levin, 2011; Guerra, Tavares, \& Araújo e Sá, 2017) fazem a mesma recomendação. Assim, o conhecimento científico gerado pelos projetos financiados (resultados e produtos) deve continuar a ser mobilizado nas diversas esferas da sociedade (ex. política, institucional, empresarial, educativa), mesmo após o término do financiamento da investigação, e pode contribuir para informar e direcionar o desenho das políticas de formação e investigação, em particular no que se refere ao desenvolvimento e sustentabilidade de inovações educativas (Guerra, 2021).

Do exposto, emergem três ideias-chave:

1 - as inovações educativas desenvolvidas no contexto do ensino superior, em particular resultante de investigação financiada, podem (e devem) contribuir para a transformação das práticas académicas dos sujeitos envolvidos (ex. docentes e estudantes);

2 - a sustentabilidade da investigação depende de vários fatores, tais como a continuidade do financiamento dos projetos, o apoio da instituição de acolhimento e as dinâmicas de trabalho dos elementos da equipa dos projetos (Guerra, 2021); 
3 - o conhecimento científico produzido no âmbito dessa investigação pode (e deve) ser disseminado e mobilizado (de formas diversas) na comunidade (ex. académica, científica, política, sociedade em geral), para informar o desenho de políticas de formação e investigação neste âmbito.

\section{A metodologia de investigação}

A investigação está enquadrada no paradigma sócio-crítico de natureza qualitativa, com caráter transformador, focada na determinação dos significados das ações individuais e das interações sociais a partir da perspetiva dos atores intervenientes no processo (Amado, 2017; Creswell, \& Clark, 2004; Coutinho, 2014).

A investigação é do tipo Investigação \& Desenvolvimento (I\&D)- também conhecida como Design-Based Research (Barab \& Squire, 2004). A opção pelo I\&D emerge de três razões:

1) é uma forma inovadora e criativa para encontrar soluções para as problemáticas educacionais identificadas nos projetos, respondendo a questões complexas (ex. sociais, educativas, políticas) com vista a, em última instância, contribuir para uma alteração (ex. na prática educativa) que seja positiva, significativa e sustentável.

2) é utilizada em contextos investigativos da área do design (Design-Based Research Collective, 2003), onde se procura perceber as relações entre teoria, o artefato projetado e a prática e que, nos últimos anos, tem sido adotada pela autora no seu percurso investigativo realizado no âmbito do Mestrado (Guerra, 2007) e Doutoramento (Guerra, 2012).

3) assume a abordagem design thinking (Riverdale \& Ideo, 2012) para o desenvolvimento do artefato - o rSIE - adaptando princípios do modelo de design centrado na atividade (Gay \& Hembrooke, 2004).

O processo de I\&D passa por cinco momentos interativos e iterativos:

1) Desvendar (discovery) - identificação da problemática e contexto do estudo, elencando as questões de investigação a que se pretendeu dar resposta com a realização do estudo.

2) Compreender (interpretation) - revisão de literatura onde se procura inspiração em fontes de informação diversas, apresentando conceptualmente o significado de sustentabilidade de investigação em educação e fatores da sua promoção e/ou inibição, em particular no ensino superior.

3) Arquitetar (ideation) - design do referencial, apresentando os participantes envolvidos, os procedimentos metodológicos seguidos, e as técnicas e instrumentos usados para a recolha e análise de dados para avaliação da qualidade dos protótipos desenvolvidos. 
4) Experimentar (experimention) - testagem de ideias integradas no referencial em contexto real e, ainda, a avaliação da sua eficácia numa permanente interação e iteração do feedback gerado pela mesma.

5) Evolucionar (evolution) - partilha e discussão dos resultados gerados, retirando-se conclusões e tecidas considerações finais, servindo de estímulo e orientação para se promover a sustentabilidade da investigação em educação.

\section{Resultados (in)tangíveis do projeto}

Tratando-se de um trabalho que começou com uma 'ideia' e culminou em diversos resultados (in)tangíveis, a metodologia I\&D foi a solução adequada para desenvolver e partilhar os processos, produtos e implicações do projeto de investigação.

\section{1 - Conceptualização de sustentabilidade da investigação}

A revisão de literatura (02/2019 a 01/2021) permitiu identificar referenciais teóricos e metodológicos sobre a sustentabilidade da investigação educacional centrada na inovação educativa no ensino superior. Este processo permitiu o aprofundamento da revisão narrativa de literatura (Guerra \& Costa, 2016), integrando numa nova publicação internacional uma sistematização do conhecimento científico produzido na área em que se insere o estudo , bem como a sua extensão através do contributo de investigadores da área. Um dos principais resultados desta fase foi o livro "Sustainable Pedagogical Research in Higher Education: The Political, Institutional and Financial Challenges", da editora Routledge (Guerra, Franco e Seabra (2021). Considerando as possibilidades teóricas e práticas da sustentabilidade da investigação no ensino superior, em que o financiamento dos projetos é muitas vezes difícil de garantir, este livro compartilha os resultados de diversas experiências e reflexões de académicos e líderes institucionais do ensino superior sobre formas de promover e sustentar a inovação pedagógica no contexto do ensino superior.

\section{2 - Desenvolvimento do referencial}

O estudo empírico tem implicado o desenho, implementação e avaliação de estratégias e instrumentos a integrar no rSIE a partir: do lançamento de bases conceptuais e metodológicas para a criação de uma rede de atores-chave da investigação em educação, com vista à reflexãoação sobre a sustentabilidade da investigação centrada na promoção da inovação educativa no Ensino Superior; da organização de uma comunidade de prática $(\mathrm{Cp})$ envolvendo pessoas dedicadas ao desenvolvimento de projetos de investigação em educação, no sentido de 
potenciarem a mobilização do conhecimento científico nas diversas esferas da sociedade (política, educacional, empresarial).

Assim, e desde a sua génese, o projeto beneficia da colaboração de diversos atores-chave do ensino superior (ex., docentes e estudantes, investigadores em Ciências da Educação, Ciências Naturais e Engenharia) no desenho de estratégias e instrumentos promotores da mobilização do conhecimento científico em estreita articulação entre o ensino, a investigação e a extensão com a sociedade. Os atores-chave, pelas suas responsabilidades institucionais, conhecimento científico e/ou motivações académicas e pessoais, têm colaborado com a autora deste artigo na procura de um entendimento mais aprofundado sobre o desenvolvimento e a sustentabilidade da investigação centrada na inovação educativa no ensino universitário.

Elencam-se, a título de exemplo, alguns dos projetos desenvolvidos desde fevereiro de 2019:

- produção da primeira versão do referencial com estratégias para o desenvolvimento e sustentabilidade de inovações educativas no contexto universitário português. O referencial foi produzido a partir da articulação entre os resultados obtidos com o primeiro estudo de pósdoutoramento (Guerra \& Costa, 2016) e (novas) conceptualizações teóricas resultantes da revisão de literatura realizada na Fase I de investigação deste projeto (Guerra, 2021).

- validação das estratégias inscritas no referencial envolvendo diferentes agentes-chave (docentes e estudantes do ensino superior), recolhendo dados a partir de inquérito por questionário online.

Os resultados obtidos com a avaliação do impacte das estratégias implementadas em contexto universitário têm sido disseminados pelos próprios participantes da $\mathrm{Cp}$, procurando mobilizar o conhecimento científico produzido na comunidade científica, académica e/ou política (Guerra, Moreira, Loureiro, \& Cabrita, 2020; Huet, Guerra, da Silva Lopes, aceite).

\section{3 - Avaliação do referencial}

A análise de conteúdo dos documentos produzidos pelos participantes (ex. artigos científicos produzidos) e a análise dos registos de observação do trabalho desenvolvido na rede (observação participante da investigadora do estudo) é um processo contínuo, com ciclos de reflexão-ação para ajudar a identificar as potencialidades, constrangimentos e definir melhorias para produzir a segunda versão do referencial.

Numa fase posterior será realizado um inquérito por entrevista (possivelmente, do tipo grupo de enfoque) aos participantes para: identificar as potencialidades, constrangimentos e sugestões para a melhoria do referencial; averiguar o papel que as inovações educativas, 
desenvolvidas a partir de investigação financiada, tem (ou deve ter) na valorização das vertentes de Ensino e Investigação do trabalho académico, identificando estratégias para a sua operacionalização. No seguimento da análise de conteúdo das transcrições das entrevistas realizadas será produzida a terceira versão do referencial.

\section{Reflexão final}

O projeto relatado ao longo deste artigo resulta da continuidade do trabalho de investigação, iniciado em 2012 pelos orientadores científicos da autora deste artigo (ver Introdução), mas cujo tema de investigação continua a proporcionar curiosidade científica. Hoje, volvidos (quase) dez anos em que se estudou sobre o processo de inovação educativa no ensino superior resultante da investigação em educação, é natural afirmar que refletir sobre a multiplicidade das dimensões e processos que concorrem para a sustentabilidade da investigação foi (e continua a ser) relevante a nível científico (porque se continua a procurar respostas às questões de investigação delineadas), sendo ainda mais relevante a título pessoal, enquanto investigadora em educação.

O tema continua atual, pertinente e audacioso, mas tem que ser encarado como um processo que está em contínua transformação. Neste quadro, trata-se de um empreendimento crucial para a área da investigação em educação, devendo fomentar a precisão e validade dos seus processos metodológicos, logo, o impacte dos seus produtos.

A promoção da sustentabilidade dos projetos, após o término do período de financiamento, não pode ser encarada, apenas como um desejo abstrato, mas também, uma responsabilidade social da investigação em educação.

As agências de financiamento nacionais e europeias solicitam esforços pra a promoção da sustentabilidade da investigação financiada, fazendo-se sentir a sua necessidade no meio académico, sendo por isso um aspecto crucial nos dias que correm e nas sociedades em que vivemos, sendo por isso necessário dedicar esforços à sua compreensão e promoção na comunidade cientifica.

Não obstante, será que a comunidade científica da investigação em educação, particularmente em Portugal, está encetar 'esforços' no âmbito dos seus projetos financiados para garantir que os resultados da investigação sejam, efetivamente, promovidos pelos seus atores-chave no 'terreno' (tais como políticos, líderes institucionais, investigadores, e demais agentes educativos como professores e estudantes) de uma forma deliberada, explícita, sistemática? 
Esta interrogação afigurou-se como uma problemática particularmente relevante a fazer num contexto que advoga, entre outros princípios, a reflexão de Watt (2021) quando refere que

It is possible to present an argument that sustainable funding and influential research are vital for the continued growth of higher education policy and pedagogy and more broadly for society, political arenas, and economic interests. Wider and clearer dissemination of funded educational research and peer-reviewed findings is key especially as end-users are seeking confirmation, searching for new information, and/or pursuing possibilities for philanthropic initiatives. Additionally, evidence acquired through quality educational research is wanted, especially given the ongoing personal choice movement for all levels of education. (p.24)

Explica-se, desta forma, a relevância do projeto levado a cabo, que enfatiza um conjunto de três objetivos que é preciso procurar concretizar na comunidade científica:

1) Garantir que as instituições de ensino superior (em Portugal) assumam a relevância da sustentabilidade da investigação em educação, bem como a urgência de ser efetivamente integrada nas agendas institucionais e nas práticas da comunidade, e não apenas em manifestos institucionais;

2) Sensibilizar, orientar e apoiar os atores-chave no 'terreno' (por exemplo, líderes institucionais, docentes e estudantes) a acolherem os resultados da investigação em educação nas suas práticas, e a cultivarem, de forma deliberada, explícita e sistemática, uma inovação em prol da promoção da articulação entre investigação, formação e extensão a sociedade;

3) Promover o desenvolvimento de projetos de investigação em educação, não apenas para favorecer o desempenho académico dos participantes envolvidos (ex. estudantes, docentes) e, talvez mais importante, para que estes assumam um papel reflexivo, ativo, informado e ético nas suas vidas.

A realização deste projeto emerge, assim, como uma oportunidade para ajudar a refletir sobre o modo como a investigação em educação, particularmente a realização no contexto do ensino superior, possa ser (mais) um contributo para a sustentabilidade da investigação (no seu entendimento alargado).

Será possível, neste momento, ser investigador sem assumir um compromisso para a promoção da sustentabilidade dos resultados alcançados com a investigação?

E enquanto comunidade de investigadores em educação, não será nossa a responsabilidade social de procurar estratégias e instrumentos que mobilizem o conhecimento científico gerado nas várias esferas da sociedade?

Em última instância, dedica-se esta reflexão à comunidade científica para benefício próprio, desde logo, de uma maior discussão sobre 'novas' formas de investigar em educação. 
Agradecimentos: Este trabalho foi financiado através do Fundo Social Europeu e Programa Operacional de Capital Humano por Fundos Nacionais através da FCT, no âmbito dos projetos UIDB/00194/2020, UIDB/00073/2020 e UIDP/00073/2020.

\section{REFERENCIAS}

Amado, J. (2017). Manual de Investigação Qualitativa em Educação $3^{\text {a }}$ edição. Imprensa da Universidade de Coimbra/Coimbra University Press.

Barab, S., \& Squire, K. (2004). Design-based research: Putting a stake in the ground. The journal of the learning sciences, 13(1), 1-14.

Becan, J. E., Bartkowski, J. P., Knight, D. K., Wiley, T. R. A., DiClemente, R., Ducharme, L., \& Hiller, M. (2018). A model for rigorously applying the Exploration, Preparation, Implementation, Sustainment (EPIS) framework in the design and measurement of a large scale collaborative multi-site study. Health \& Justice, 6(1), 9. DOI: https://doi. org/10.1186/s40352-018-0068-3

Bennet, A., Bennet, D., Fafard, K., Fonda, M., Lomond, T., Messier, L., \& Vaugeois, N. (2007). Knowledge mobilization in the social sciences and humanities. Frost, WV: Mqi Press. ISBN: 9780979845932

Bogdan, R., \& Biklen, S. (1994). Investigação qualitativa em educação: uma introdução à teoria e aos métodos. Porto editora.

Boshier, R. (2009). Why is the scholarship of teaching and learning such a hard sell? Higher Education Research \& Development, 28(1), 1-15. DOI: https://doi.org/10.1080/07294360802444321

Cain, T., \& Allan, D. (2017). The invisible impact of educational research. Oxford Review of Education, 43(6), 718-732. DOI: https://doi.org/10.1080/03054985.2017.1316252

Costa, N., \& Guerra, C. (2020). Contribution of the Book for Future Directions to Advance the Sustainability of Pedagogical Research and Practice. Sustainable Pedagogical Research in Higher Education: The Political, Institutional and Financial Challenges, 113.

Coutinho, C. P. (2014). Metodologia de investigação em ciências sociais e humanas. Leya.

Coutinho, C. P. (2014). Metodologia de investigação em ciências sociais e humanas. Leya.

Creswell, J. W., \& Clark, V. L. P. (2004). Principles of qualitative research: Designing a qualitative study. Office of Qualitative \& Mixed Methods Research, University of Nebraska, Lincoln.

Creswell, J. W., Hanson, W. E., Clark Plano, V. L., \& Morales, A. (2007). Qualitative research designs: Selection and implementation. The counseling psychologist, 35(2), 236-264.

Del Pino, J. C., \& Cachapuz, A. F. (2008). En busca del perfeccionamiento de una asignatura de introducción a la química en la enseñanza universitaria. Educación química, 19(4), 332-337. 
Design-Based Research Collective. (2003). Design-based research: An emerging paradigm for educational inquiry. Educational researcher, 32(1), 5-8.

European Commission. (2014). Report to the European Commission on New modes of learning and teaching in higher education. European Comission. Retrieved from http://ec.europa.eu/education/library/reports/modernisation_en.pdf

European Commission. (2020). Erasmus+ Programme Guide 2020. Brussels, Belgium: European Commission.

Faber, N., Jorna, R., \& Van Engelen, J. O. (2009). The sustainability of "sustainability" - A study into the conceptual foundations of the notion of "sustainability." In W. R. Sheate (Ed.), Tools, techniques and approaches for sustainability: Collected writings in environmental assessment policy and management (pp. 337-369). London: World Scientific. DOI: https:// www.worldscientific.com/doi/abs/10.1142/9789814289696_0016

Fishman, B., Penuel, W. R., Hegedus, S., \& Roschelle, J. (2011). What happens when the research ends? Factors related to the sustainability of a technology-infused mathematics curriculum. Journal of Computers in Mathematics and Science Teaching, 30(4), 329-353.

Gay, G., \& Hembrooke, H. (2004). Activity-centered design: An ecological approach to designing smart tools and usable systems. Mit Press.

Guerra, C. (2007). Avaliação do storyboard e da metodologia de desenvolvimento do Courseware Sere. Mestrado, Departamento de Didáctica e Tecnologia Educativa, Universidade de Aveiro, Aveiro.

Guerra, C. (2012). Formação de professores de ciências para o uso de tecnologias (Doctoral dissertation, Tese de Doutoramento não publicada. Universidade de Aveiro: Departamento de Educação e Departamento de Comunicação e Arte).

Guerra, C. (2021). Educational research sustainability in higher education: Reflections on the concept, factors and actions for its enhancement. C. Guerra, A. Franco \& M. Seabra (Eds.), Sustainable Pedagogical Research in Higher Education: The Political, Institutional and Financial Challenges (pp.7-19). Routledge. https://www.routledge.com/Sustainable-Pedagogical-Research-in-Higher-EducationThe-Political-Institutional/Guerra-Franco-Seabra/p/book/9780367436087

Guerra, C. V., Moreira, F., Loureiro, M. J., \& Cabrita, I. (2020). Programação tangível para a inclusão e promoção das STEM. APEduC Revista-Investigação e Práticas em Educação em Ciências, Matemática e Tecnologia, 1(1), 100-114.

Guerra, C., \& Costa, N. (2016). Sustentabilidade da investigação educacional: contributos da literatura sobre o conceito, fatores e ações. Revista Lusófona de Educação, 34(34), 13 25 .

Guerra, C., \& Costa, N. (2016a). Sustentabilidade da investigação educacional: contributos da literatura sobre o conceito, fatores e ações. Revista Lusófona de Educação, 34(34), 13 25 .

Guerra, C., \& Costa, N. (2016b). Sustainability of educational research centered on innovation in Portuguese Higher Education Institutions. In Oostdam, R. J., Griffioen, D. 
M. E., Teelken, C., \& Kyndt, E. (Eds.). (2016). The Higher Education Conference 2016. Programme Book \& Book of Abstracts. decision making, 66(5), 603-618.

Guerra, C., \& Costa, N. (2018). Educational innovations in engineering education: Sustainability of funded projects developed in Portuguese higher education institutions. In 2018 3rd International Conference of the Portuguese Society for Engineering Education (CISPEE) (pp. 1-6). IEEE.

Guerra, C., Correia, A., \& Pedrosa-de-Jesus, H. (2014). Estratégias inovadoras de ensino e feedback potenciadas pelas tecnologias. O "caso" da Microbiologia da Universidade de Aveiro. Indagatio Didactica, 6(1), 292-311.

Guerra, C., Franco, A., \& Seabra, M. (2018). IAPHE'18, innovative academic practices in higher education-How to make them sustainable: International seminar e-book. Aveiro: UA Editora

Guerra, C., Franco, A., \& Seabra, M. (Eds.) (2021). Sustainable Pedagogical Research in Higher Education: The Political, Institutional and Financial Challenges. Routledge. https://www.routledge.com/Sustainable-Pedagogical-Research-in-Higher-EducationThe-Political-Institutional/Guerra-Franco-Seabra/p/book/9780367436087

Guerra, C., Franco, A., \& Seabra, M. (Eds.). (2020). Sustainable Pedagogical Research in Higher Education: The Political, Institutional and Financial Challenges. Routledge.

Guerra, C., Pedrosa-de-Jesus, H., Correia, A., Cunha, A., Almeida, A., \& Watts, M. (2015). Promoting Academic Development trough situated critical reflection. In Transitions in Teacher Education and Professional Identities-Proceedings of the ATEE Annual Conference (pp. 267-278). Braga: University of Minho.

Guerra, C., Tavares, R., \& Araújo e Sá, M. H. (2017). SCoRE'17: Science communication for researchers in education: Autumn school e-book.. ISBN: 978-972-789-526-7

Guerra, C.; Loureiro, M.J.; Senos, S. (in press). "Didactic, Technological and Social challenges in Portuguese higher education during the pandemic confinement: testimonials of experienced educational trainers". In Developing a Post-Pandemic Paradigm for Virtual Technologies in Higher Education, edited by Sandra Loureiro; João Guerreiro. PA, United States: IGI Global Publisher of Timely Knowledge.

Huet, I; Guerra, C.; Da Silva Lopes, B (in press). "Undergraduate Research in Portugal. Section IV - International Perspective / Countries". In International Handbook on Undergraduate Research: Concepts for and Experiences with Student Research, edited by Harald A. Mieg; Elizabeth Ambos; Angela Brew; Wolfgang Deicke; Susanne Haberstroh; Judith Lehmann. Londo, United Kingdom: d. Mieg / Ambos / Brew / Galli / Lehmann.

Hutchings, P., Huber, M. T., \& Ciccone, A. (2011). Why the scholarship of teaching and learning matters today. In P. Hutchings, M. T. Huber, \& A. Ciccone (Eds.), The scholarship of teaching and learning reconsidered: Institutional integration and impact (pp. 1-23). San Francisco: Jossey-Bass

Laksov, K. B. (2019). Lessons learned: Towards a framework for integration of theory and practice in academic development. International Journal for Academic Development, 24(4), 369-380. DOI: https://doi.org/10.1080/1360144X.2018.1549046 
Levin, B. (2011). Mobilising research knowledge in education. London Review of Education, 9(1), 15-26. DOI: https://doi.org/10.1080/14748460.2011.550431

Loh, L. C., Friedman, S. R., \& Burdick, W. P. (2013). Factors promoting sustainability of education innovations: A comparison of faculty perceptions and existing frameworks. Education for Health, 26(1), 32.

Neuendorf, K. A. (2018). 18 Content analysis and thematic analysis. Advanced Research Methods for Applied Psychology: Design, Analysis and Reporting, 211.

Pedrosa-de-Jesus, H., Guerra, C., \& Watts, M. (2017). University teachers' self-reflection on their academic growth. Professional development in education, 43(3), 454-473.

Pedrosa-de-Jesus, H., Guerra, C., \& Watts, M. (2018). Models of Teachers' SoTL. In Academic Growth in Higher Education (pp. 186-199). Brill Sense.

Pedrosa-de-Jesus, H., Guerra, C., \& Watts, M. (2019, October). Active co-constructive written feedback: Promoting students' critical thinking in a higher education context. In Proceedings of the Seventh International Conference on Technological Ecosystems for Enhancing Multiculturality (pp. 129-136).

Pedrosa-de-Jesus, H., Moreira, A. C., da Silva Lopes, B., Guerra, C., \& Watts, M. (2018). Assessment and Feedback. In Academic Growth in Higher Education (pp. 200-216). Brill Sense.

Pedrosa-de-Jesus, M. H., Correia, A., Gonçalves, F., Almeida, A., Cunha, A., Moreira, A., da Silva Lopes, B., Guerra, C., \& Watts, M. (2015). Academic development in Higher Education through innovative approaches in teaching, assessment and feedback. research@ua, 6, 36-36.

Ribeiro, J. M., Guerra, C., \& Sá, P. (2016). Neri de Souza, F.; Neri de Souza, D. \& Costa, AP (Orgs.).(2015). Investigação qualitativa: inovação, dilemas e desaĺos. Volume 2. Aveiro: Ed. Ludomedia (170 pág). Revista Lusófona de Educação, (34), 211-214.

Riverdale \& Ideo (2012). Design Thinking for educators (2nd ed.). Retrieved from http://www. designthinkingforeducators.com/toolkit/

Sarriot, E., Ricca, J., Yourkavitch, J., \& Ryan, L. (2008). Sustained Health Outcomes (SHOUT) group. Taking the long view: A practical guide to sustainability planning and measurement in community-oriented health programming. Calverton, UK: Macro International Inc.

Savaya, R., Elsworth, G., \& Rogers, P. (2009). Projected sustainability of innovative social programs. Evaluation Review, 33(2), 189-205. DOI:

https://doi.org/10.1177/0193841X08322860

Scheirer, M. A. (2005). Is sustainability possible? A review and commentary on empirical studies of program sustainability. American Journal of Evaluation, 26(3), 320-347. DOI: https://doi.org/10.1177/1098214005278752

Scheirer, M. A. (2013). Linking sustainability research to intervention types. American Journal of Public Health, 103(4), e73-e80. DOI: https://doi.org/10.2105/AJPH.2012.300976 
Scheirer, M. A., \& Dearing, J. W. (2011). An agenda for research on the sustainability of public health programs. American Journal of Public Health, 101(11), 2059-2067. DOI: https://doi. org/10.2105/AJPH.2011.300193

Schell, S. F., Luke, D. A., Schooley, M. W., Elliott, M. B., Herbers, S. H., Mueller, N. B., \& Bunger, A. C. (2013). Public health program capacity for sustainability: A new framework. Implementation Science, 8(1), 15. DOI: https://doi.org/10.1186/1748-59088-15

Shediac-Rizkallah, M. C., \& Bone, L. R. (1998). Planning for the sustainability of community-based health programs: Conceptual frameworks and future directions for research, practice and policy. Health Education Research, 13(1), 87-108. DOI: https://doi.org/10.1093/her/13.1.87

\section{SOBRE A AUTORA:}

\section{Cecília Guerra}

Doutora em Multimédia em Educação pela Universidade de Aveiro. Investigadora da Universidade de Aveiro, no Departamento de Educação de Psicologia. Integra o Centro de Investigação em Didática e Tecnologia na Formação de Formadores da Universidade de Aveiro. E-mail: cguerra@ua.pt

iD https://orcid.org/0000-0002-2560-165X

\section{ANEXO \\ REFERENCIAL}

\section{Referencial de Sustentabilidade da Investigação em Educação (rSIE) - primeira versão}

\begin{tabular}{l} 
A - Relevância dos projetos de investigação \\
\hline a) para diferentes sistemas e atores do ensino superior a diferentes níveis de atuação do ensino \\
\hline a1) A nível/níveis MACRO (ex.: agências de financiamento da investigação, sistema central definidor de \\
políticas educativas e os respetivos representantes) \\
a2) A nível/níveis MESO (ex.: universidades, escolas, empresas e outras instituições e seus representantes, \\
onde os resultados da investigação foi realizada ou pode ser utilizada) \\
\hline a3) A nível/níveis MICRO (pessoas enquanto indivíduos promotores de ação e/ou I\&D: docentes \\
universitários, professores da escola, empresários, investigação/coordenação de projetos, alunos, pais, ...) \\
\hline B - Consistência de percursos metodológicos \\
\hline b) A investigação é apresentada na continuidade de estudos prévios \\
\hline c) Integra ações concretas para avaliar os resultados do/da estudo/ investigação \\
\hline d) Refere ações concretas para Disseminar, Explorar e/ou Comunicar os resultados (da investigação) \\
\hline C - Impacte da investigação \\
\hline g) Os recursos educativos (guiões, sequências didáticas, aplicações, etc.) encontram-se em acesso aberto \\
\hline h) As atividades científicas convergem para o desenvolvimento profissional de diferentes atores \\
\hline i) ...
\end{tabular}

Recebido em: 31 de maio de 2021

Aprovado em: 22 de junho de 2021

Publicado em: 01 de setembro de 2021 